\title{
Fast magnetohydrodynamic oscillations in a force-free line-tied coronal arcade
}

\author{
A. J. Díaz ${ }^{1}$, T. Zaqarashvilii ${ }^{2}$, and B. Roberts ${ }^{1}$ \\ 1 Mathematical Institute, University of St Andrews, St Andrews, KY16 9SS, Scotland, UK \\ e-mail: [antonio; bernie] @mcs.st-and.ac.uk \\ 2 Departament de Física, Universitat de les Illes Balears, 07122 Palma de Mallorca, Spain \\ e-mail: temury.zaqarashvili@uib.es
}

Received 27 October 2005 / Accepted 28 April 2006

ABSTRACT

\begin{abstract}
Aims. We discuss a simple model of a line-tied coronal arcade with piecewise constant density to explore the effects of curvature on radially polarised fast modes.

Methods. A partial differential equation is derived for the velocity perturbation of the fast modes and it is solved analytically in terms of Bessel functions of half integer order, obtaining a dispersion relation.

Results. The properties of the modes are studied in terms of the parameters. All the modes are leaky under these conditions. Besides the usual kink and sausage modes, new families are described: the vertical, swaying (longitudinal), and rocking modes arise.

Conclusions. The damping rates are similar to observed rates. For thin arcades the modes are markedly different from those of a straight slab and resemble more the modes of a circular membrane.
\end{abstract}

Key words. Sun: oscillations - Sun: magnetic fields - Sun: corona

\section{Introduction}

Observations show clearly that the solar corona is structured in regions with open magnetic fields or coronal holes and in regions with closed magnetic field (with field endpoints anchored in the photosphere). Closed regions are composed of myriads of coronal loops, sometimes aligned together forming arcades with a wide range of densities and temperatures. Oscillations are observed in these coronal structures, especially in loops (see the reviews in Aschwanden 2004 and Nakariakov \& Verwichte 2005) and also in coronal arcades (Verwichte et al. 2004, 2005)

The oscillatory properties of an isolated, infinite, uniform, cylindrically-symmetric flux tube were discussed by Edwin \& Roberts (1983) and Roberts et al. (1984). These works provide a basic theory for understanding the oscillations of coronal loops, and their results are used to extract information regarding the modes (coronal seismology). In particular, certain modes are known to be highly dispersive and different families of modes are supported by the flux tube: kink or sausage modes, depending of the azimuthal wavenumber, or body and surface modes, depending on the radial wavenumber, may arise. However, it is not known how these relatively simple models are affected by the complexities of the real corona. Most theoretical studies of coronal oscillations tend to study straight field configurations, exploring a variety of aspects; for example, the effect of structure along the axis has attracted recent interest (Díaz et al. 2004; Van Doorsselaere et al. 2004; Andries et al. 2005a,b; Donnelly et al. 2006; McEwan et al. 2006).

Regarding the curvature of magnetic fields, previous numerical studies have explored several aspects. Smith et al. (1997) studied a potential arcade with an exponential equilibrium density profile and estimated the damping times of the stationary modes. Brady \& Arber (2005) and Selwa et al. (2005) explored numerically the effect of excitation in the footpoints, and they also pointed out that some of the features described in Smith et al. (1997) are due to computation artifacts. All these works show that leakage seems to be an important effect resulting from curvature.

Recently, Verwichte et al. (2005) studied an equilibrium in which the plasma density depended on the radial variable $r$ as $r^{-4}$, and they described analytically the resulting properties of the modes of an arcade. They point out that for less pronounced profiles all the modes are oscillatory in the coronal region (and therefore leaky).

All these studies raise questions about the role of magnetic curvature on the modes of oscillation. Following Verwichte et al. (2005), we consider here a simple model of a potential field that varies in strength with the radial coordinate $r$; the plasma density is taken to be piecewise uniform. By studying such a case, we are able to shed light on the role of an arcade structure in the modes of oscillation of a dense loop modelled by a slab geometry.

Our aim here is to discuss analytically the modes of oscillation of an arcade. In this context it is interesting to note the observational results of Wang \& Solanki (2004), who distinguish between vertical and horizontal modes. Following their lead, we find it convenient here to distinguish the several types of vibrations that may arise. We term the motion as a vertical mode if the arcade is displaced vertically (perpendicular to the photospheric base) in the oscillation. The vertical vibration may lead to a global raising or lowering of the arcade structure at its apex, which we refer to as the vertical kink mode (by analogy with the kink mode of a straight tube). Alternatively, the vertical vibration may squeeze (or rarefy) the plasma at the apex of the arcade because of unequal vertical motions of the upper and lower surfaces of the arcade; we refer to this as a vertical sausage mode. The vertical mode (kink or sausage) is symmetric about the apex 


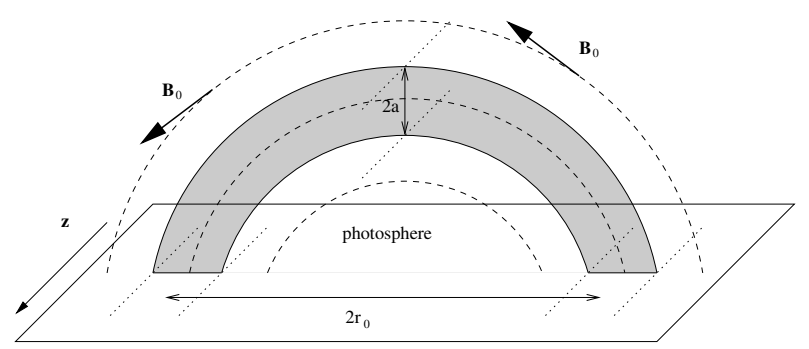

Fig. 1. Sketch of the equilibrium configuration representing a semicircular magnetic arcade of length $L=\pi r_{0}$ and width $2 a$. The dense zone between $r=r_{0}-a$ and $r=r_{0}+a$ (shaded grey) has plasma density $\rho_{\mathrm{l}}$ and the density in the coronal environment is $\rho_{\mathrm{c}}$. The magnetic field $\boldsymbol{B}_{0}$ points in the azimuthal direction. The configuration is described by cylindrical coordinates $(r, \varphi, z)$, with invariance in the $z$-direction.

of the arcade. However, the arcade vibrations may not be symmetric about the apex but instead involve sideways vibrations in a direction parallel to the photosphere, so that the arcade sways along the field first towards one footpoint and then towards the other footpoint; we refer to this as the swaying mode. Finally, the whole arcade may vibrate by rocking horizontally along the central axis of the arcade, corresponding to a motion in the longitudinal direction of the arcade; we term this the rocking mode. In terms of a straight flux tube, the rocking mode is a kink vibration, but in an arcade (and loop) structure it is more informative to label its motion distinctively from the vertical mode. The rocking mode requires a separate investigation to the vertical and swaying modes explored here and so will be left for a future investigation. A comment about this terminology is in order. As we have noted, in this particular problem the notation introduced for the different types of modes relates naturally with the longitudinal harmonics of a straight slab or tube (Erwin \& Roberts 1982, 1983): the vertical mode corresponds to oscillations with one extremum in the direction along the magnetic field, while the swaying modes corresponds to modes with two extrema and a node in the apex. However, this connection cannot be drawn for geometrically more complex configurations, while the classification of the modes we introduce should still remain valid. Moreover, our description of the modes in this way is perhaps more natural from the observational point of view and this may therefore help in the interpretation of observations.

\section{Equilibrium model and wave equations}

The equilibrium model consists of a curved arcade of length $L=\pi r_{0}$ filled with an uniform plasma of density $\rho_{\mathrm{l}}$, which is embedded in coronal material of uniform density $\rho_{\mathrm{c}}$. The plasma is permeated by a force-free magnetic field of the form

$\boldsymbol{B}_{0}=B_{\varphi}(r) \boldsymbol{e}_{\varphi}=B_{0} \frac{r_{0}}{r} \boldsymbol{e}_{\varphi}$,

satisfying $\nabla \cdot \boldsymbol{B}_{0}=0$ and $\nabla \times \boldsymbol{B}_{0}=0$. The whole structure is invariant in the $z$-direction (along the arcade) and there is no explicit dependence of the equilibrium quantities on the $z$ and $\varphi$ coordinates. This model has also been recently discussed by Verwichte et al. (2005). A sketch of the model is presented in Fig. 1.

The starting point for our MHD wave analysis are the linearised ideal MHD equations for a zero- $\beta$ plasma,

$\rho_{0} \frac{\partial \boldsymbol{v}}{\partial t}=\frac{1}{\mu}(\nabla \times \boldsymbol{B}) \times \boldsymbol{B}_{0}+\frac{1}{\mu}\left(\nabla \times \boldsymbol{B}_{0}\right) \times \boldsymbol{B}$, $\frac{\partial \boldsymbol{B}}{\partial t}=\nabla \times\left(\boldsymbol{v} \times \boldsymbol{B}_{0}\right)$,

$\nabla \cdot \boldsymbol{B}=0$.

We name the equilibrium density as $\rho_{0}$, which is a constant that can take the values of $\rho_{\mathrm{l}}$ or $\rho_{\mathrm{c}}$. These equations describe the behaviour of the perturbed magnetic field $\boldsymbol{B}$, velocity $\boldsymbol{v}$, from which the perturbed density $\rho$ can be obtained. In a zero- $\beta$ plasma all oscillations are purely a response to magnetic forces; in a uniform medium, these modes would be the Alfvén and fast magnetoacoustic waves.

The equation of motion (Eq. (2)) leads to

$\mu \rho_{0} \frac{\partial^{2} \boldsymbol{v}}{\partial t^{2}}=-\boldsymbol{B}_{0} \times\left(\nabla \times \frac{\partial \boldsymbol{B}}{\partial t}\right)$,

in which $\nabla \times \boldsymbol{B}_{0}=0$ has been applied. Using the induction equation (Eq. (3)) we obtain

$$
\begin{array}{r}
\frac{\partial \boldsymbol{B}}{\partial t}=\left(\frac{B_{\varphi}}{r} \frac{\partial v_{r}}{\partial \varphi}\right) \boldsymbol{e}_{r}-\left(v_{r} \frac{\partial B_{\varphi}}{\partial r}+B_{\varphi} \frac{\partial v_{r}}{\partial r}\right) \boldsymbol{e}_{\varphi} \\
+\left(\frac{B_{\varphi}}{r} \frac{\partial v_{z}}{\partial \varphi}\right) \boldsymbol{e}_{z}
\end{array}
$$

Substituting this result into Eq. (5) we obtain (after some algebra) the following differential equations for the components of the perturbed velocity $\boldsymbol{v}=\left(v_{r}, v_{\varphi}, v_{z}\right)$ :

$$
\begin{aligned}
& \frac{\partial^{2} v_{r}}{\partial t^{2}}-c_{\mathrm{A}}^{2}(r)\left[\frac{\partial^{2} v_{r}}{\partial r^{2}}-\frac{1}{r} \frac{\partial v_{r}}{\partial r}+\frac{v_{r}}{r^{2}}+\frac{1}{r^{2}} \frac{\partial^{2} v_{r}}{\partial \varphi^{2}}\right]=0 \\
& \frac{\partial^{2} v_{\varphi}}{\partial t^{2}}=0 \\
& \frac{\partial^{2} v_{z}}{\partial t^{2}}-c_{\mathrm{A}}^{2}(r) \frac{1}{r^{2}} \frac{\partial^{2} v_{z}}{\partial \varphi^{2}}=0
\end{aligned}
$$

where $c_{\mathrm{A}}^{2}(r)$ is the squared Alfvén speed,

$c_{\mathrm{A}}^{2}(r)=\frac{B_{\varphi}^{2}}{\mu \rho_{0}}=\frac{B_{0}^{2}}{\mu \rho_{0}}\left(\frac{r_{0}}{r}\right)^{2}=c_{\mathrm{A} 0}^{2}\left(\frac{r_{0}}{r}\right)^{2}$.

Here $c_{\mathrm{A} 0}=B_{0} / \sqrt{\mu \rho_{0}}$ denotes a constant value for the Alfvén speed, since the equilibrium density $\rho_{0}$ as constant in both the arcade and the coronal medium.

Thus, $v_{\varphi}=0$ from Eq. (8). Equation (9) represents an Alfvén wave polarised in the $z$-direction, with each field line oscillating at its own local Alfvén frequency $\omega_{\mathrm{A}}=m c_{\mathrm{A}}(r) / r$,

$v_{z}(r, \varphi, t)=v_{z 0} \operatorname{expi}\left(\omega_{\mathrm{A}}(r) t+m \varphi\right)$.

Such Alfvénic oscillations and the coupling with the fast modes have been discussed in the context of magnetospheric oscillations (Allan et al. 1986a,b; Lee \& Lysak 1989; Wright 1992, and references therein) and in the consideration of phase mixing (Heyvaerts \& Priest 1983). If propagation along the $z$-axis is permitted, then the Alfvén and fast modes become coupled; but here we can concentrate on the fast mode separately, setting $v_{z}=0$.

To describe the radial motion $v_{r}$ we Fourier analyse in time $t$ and angle $\varphi$, writing

$v_{r}(r, \varphi, t)=\bar{v}_{r}(r) \operatorname{expi}(\omega t+m \varphi)$.

Notice that Eq. (7) admits separation of variables, which leads to the Fourier decomposition in Eq. (12). Then, writing $\bar{v}_{r}=r u_{r}$ we obtain

$\frac{\mathrm{d}^{2} u_{r}}{\mathrm{~d} r^{2}}+\frac{1}{r} \frac{\mathrm{d} u_{r}}{\mathrm{~d} r}+\left(\frac{\omega^{2}}{c_{\mathrm{A}}^{2}(r)}-\frac{m^{2}}{r^{2}}\right) u_{r}=0$. 
Equation (13) may also be obtained from the Hain-Lüst equation (see, for example, Goossens 1991) applied to a purely azimuthal equilibrium magnetic field.

In the case of a uniform plasma density ( $\rho_{0}=$ constant) Eq. (13) may be reduced to a form of Bessel's equation of halfinteger order, $m / 2$, and variable $s=r \omega /\left(2 c_{\mathrm{A}}(r)\right)$. In particular, the general solution to Eq. (13) is

$$
\begin{aligned}
\bar{v}_{r}(r)=r u_{r}(r) & =A r J_{m / 2}\left(\frac{\omega}{2 c_{\mathrm{A} 0} r_{0}} r^{2}\right) \\
& +B r Y_{m / 2}\left(\frac{\omega}{2 c_{\mathrm{A} 0} r_{0}} r^{2}\right),
\end{aligned}
$$

with $A$ and $B$ arbitrary constants. We consider such solutions in conjunction with the boundary conditions

$\left[v_{r}\right]=\left[\frac{\partial v_{r}}{\partial r}\right]=0$.

These boundary conditions follow from Eq. (13) or they may be deduced from first principles (Goedbloed 1983), much as in Díaz (2004).

Additionally, the line-tying boundary condition must be enforced in both arcade footpoints $\varphi=0$ and $\varphi=\pi$, which implies that $m$ is a non-zero integer and the arbitrary phase degree of freedom in Eq. (12) is restricted. Then

$v_{r}(r, \varphi)=\bar{v}_{r}(r) \sin (m \varphi)$,

with $\bar{v}_{r}(r)$ given by Eq. (14).

\section{Analytical solution}

We apply the solution in Eq. (14) to the case when there are three regions: a dense arcade plasma in $r_{0}-a<r \leq r_{0}+a$, and coronal plasma in the surrounding regions. Then

$\bar{v}_{r}(r)=\left\{\begin{array}{cl}A_{1} r J_{m / 2}\left(k_{\mathrm{c}}(r) r\right), & r \leq r_{0}-a, \\ A_{2} r J_{m / 2}\left(k_{1}(r) r\right) & \\ \quad+A_{3} r Y_{m / 2}\left(k_{1}(r) r\right), & r_{0}-a<r \leq r_{0}+a, \\ A_{4} r H_{m / 2}^{1}\left(k_{\mathrm{c}}(r) r\right), & r>r_{0}+a .\end{array}\right.$

with $k_{\mathrm{l}}(r)$ and $k_{\mathrm{c}}(r)$ defined by

$k_{j}(r)=\frac{\omega}{2 c_{\mathrm{A}}^{j}(r)}=\frac{\omega}{2 c_{\mathrm{A} 0}^{j}} \frac{r}{r_{0}}, j=1$ or $\mathrm{c}$.

The form of the solution in the outer region, $r>r_{0}+a$, has been selected so that we have an outgoing wave as $r \rightarrow \infty$. Because the Alfvén speed declines rapidly as $r \rightarrow \infty$, no trapped waves are here possible: all modes are leaky no matter how dense the loop structure. This is in contrast with the case of a magnetic slab (Edwin \& Roberts 1982). However, for a variable density profile trapped waves are again possible (Verwichte et al. 2005). In the inner region, $r \leq r_{0}-a$, the solution in Eq. (17) has been chosen to admit only the $J$ Bessel function; this is so that $v_{r}$, together with magnetic perturbation (Eq. (6)), are bounded as $r \rightarrow 0$.

Applying the boundary conditions (Eq. (15)) the constant coefficients can be related and the following dispersion relation obtained

$$
\begin{aligned}
& \frac{Y_{m / 2}\left(\zeta_{-}^{\mathrm{l}}\right) J_{m / 2}{ }^{\prime}\left(\zeta_{-}^{\mathrm{c}}\right)-\sqrt{\rho_{1} / \rho_{\mathrm{c}}} Y_{m / 2}{ }^{\prime}\left(\zeta_{-}^{1}\right) J_{m / 2}\left(\zeta_{-}^{\mathrm{c}}\right)}{\sqrt{\rho_{1} / \rho_{\mathrm{c}}} J_{m / 2}{ }^{\prime}\left(\zeta_{-}^{1}\right) J_{m / 2}\left(\zeta_{-}^{\mathrm{c}}\right)-J_{m / 2}\left(\zeta_{-}^{1}\right) J_{m / 2}\left(\zeta_{-}^{\mathrm{c}}\right)}= \\
& \frac{Y_{m / 2}\left(\zeta_{+}^{1}\right) H_{m / 2}^{(1)}{ }^{\prime}\left(\zeta_{+}^{\mathrm{c}}\right)-\sqrt{\rho_{\mathrm{l}} / \rho_{\mathrm{c}}} Y_{m / 2}{ }^{\prime}\left(\zeta_{+}^{1}\right) H_{m / 2}^{(1)}\left(\zeta_{+}^{\mathrm{c}}\right)}{\sqrt{\rho_{1} / \rho_{\mathrm{c}}} J_{m / 2}{ }^{\prime}\left(\zeta_{+}^{1}\right) H_{m / 2}^{(1)}\left(\zeta_{+}^{\mathrm{c}}\right)-J_{m / 2}\left(\zeta_{+}^{1}\right) H_{m / 2}^{(1)}{ }^{\prime}\left(\zeta_{+}^{\mathrm{c}}\right)} .
\end{aligned}
$$

\section{Here we have written}

$\zeta_{ \pm}^{j}=\frac{\omega}{2 c_{\mathrm{A}}^{j}\left(r_{0} \pm a\right)}\left(r_{0} \pm a\right), j=1$ or $\mathrm{c}$.

and a dash (') denotes the derivative of a Bessel function. The dispersion relation in Eq. (19) is a transcendental equation for complex $\omega$.

As described earlier, there are various types of solution which here we may describe in terms of the integer $m$. If $m=1$, then the solution has an anti-node at the arcade summit; we have named this the vertical mode to distinguish it from the transversal rocking modes (with displacement in the $z$-direction in a 3D model). Modes with $m=2$ have a node at the arcade apex and appear to shift the arcade laterally; these are the swaying modes. Also notice that in this particular problem, the notation introduced for the different types of modes can be related with the longitudinal harmonics in the straight slab: the vertical ( $m=1)$ correspond to modes with one extrema in the direction along the magnetic field ( $n=1$ in Edwin \& Roberts 1982), while the swaying modes $(m=2)$ correspond to modes with two extrema and a node in the apex $(n=2$ in Edwin \& Roberts 1982), and so on. The modes may also be classified, depending on their symmetry with respect to the arcade axis, as kink (even) or sausage (odd) modes, although this classification is not evident in Eqs. (17) and (19). Families with higher values of $m$ are less relevant for the standing modes. We discuss further these features when studying the resulting spatial distributions.

\subsection{Slim arcade limit}

The solutions to Eq. (19) can be studied in the limiting case $a / L \rightarrow 0$ corresponding to a slim and long arcade. Using a Taylor expansion of the Bessel functions centred on the points $\zeta_{j}^{0}=\omega L /\left(2 \pi c_{\mathrm{A}}^{j}\right)$ and retaining only first order terms in $a / L$, we obtain (after some algebra)

$$
\begin{aligned}
& \alpha\left(Y_{m / 2}^{\prime}\left(\zeta_{1}^{0}\right) J_{m / 2}\left(\zeta_{1}^{0}\right)-Y_{m / 2}\left(\zeta_{1}^{0}\right) J_{m / 2}^{\prime}\left(\zeta_{1}^{0}\right)\right) \\
& \left(J_{m / 2}^{\prime}\left(\zeta_{\mathrm{c}}^{0}\right) H_{m / 2}^{(1)}\left(\zeta_{\mathrm{c}}^{0}\right)-J_{m / 2}\left(\zeta_{\mathrm{c}}^{0}\right) H_{m / 2}^{(1)}{ }^{\prime}\left(\zeta_{\mathrm{c}}^{0}\right)\right) \\
& +2 \frac{a}{L} \zeta_{j}^{0}\left\{-\alpha\left(Y_{m / 2}^{\prime}\left(\zeta_{1}^{0}\right) J_{m / 2}\left(\zeta_{1}^{0}\right)-Y_{m / 2}\left(\zeta_{1}^{0}\right) J_{m / 2}^{\prime}\left(\zeta_{1}^{0}\right)\right)\right. \\
& \left(J_{m / 2}^{\prime \prime}\left(\zeta_{\mathrm{c}}^{0}\right) H_{m / 2}^{(1)}\left(\zeta_{\mathrm{c}}^{0}\right)-J_{m / 2}\left(\zeta_{\mathrm{c}}^{0}\right) H_{m / 2}^{(1)}{ }^{\prime \prime}\left(\zeta_{\mathrm{c}}^{0}\right)\right) \\
& +\alpha^{2}\left(J_{m / 2}^{\prime}\left(\zeta_{\mathrm{c}}^{0}\right) H_{m / 2}^{(1)}\left(\zeta_{\mathrm{c}}^{0}\right)+J_{m / 2}\left(\zeta_{\mathrm{c}}^{0}\right) H_{m / 2}^{(1)}{ }^{\prime}\left(\zeta_{\mathrm{c}}^{0}\right)\right) \\
& \left(J_{m / 2}^{\prime \prime}\left(\zeta_{1}^{0}\right) Y_{m / 2}\left(\zeta_{1}^{0}\right)-J_{m / 2}\left(\zeta_{1}^{0}\right) Y_{m / 2}^{\prime \prime}\left(\zeta_{1}^{0}\right)\right) \\
& +\alpha^{3} 2 J_{m / 2}\left(\zeta_{\mathrm{c}}^{0}\right) H_{m / 2}^{(1)}\left(\zeta_{\mathrm{c}}^{0}\right) \\
& \left.\times\left(Y_{m / 2}^{\prime \prime}\left(\zeta_{1}^{0}\right) J_{m / 2}^{\prime}\left(\zeta_{1}^{0}\right)-Y_{m / 2}^{\prime}\left(\zeta_{1}^{0}\right) J_{m / 2}^{\prime \prime}\left(\zeta_{1}^{0}\right)\right)\right\}=0
\end{aligned}
$$

where $\alpha=\left(\rho_{1} / \rho_{\mathrm{c}}\right)^{1 / 2}$. Now we use the recursive relations of the Bessel functions and the expressions for their Wronskians (Abramowitz \& Stegun 1967) to derive the following equation

$$
\frac{\mathrm{i}}{2 \pi(a / L)\left(\rho_{1} / \rho_{\mathrm{c}}-1\right)}+\zeta_{1}^{0^{2}} J_{m / 2}\left(\zeta_{\mathrm{c}}^{0}\right) H_{m / 2}^{(1)}\left(\zeta_{\mathrm{c}}^{0}\right)=0 .
$$

Next we use the asymptotic approximation for Bessel functions of large argument, obtaining

$$
\frac{\mathrm{i}}{2(a / L)\left(\rho_{1} / \rho_{\mathrm{c}}-1\right)}+\zeta_{1}^{0}\left[\exp \left(\mathrm{i} 2\left(\zeta_{\mathrm{c}}^{0}-b\right)\right)-1\right]=0 .
$$


Finally, if we assume that the imaginary part of the frequency is larger than the real part $\left(\omega=\omega_{\text {re }}+\mathrm{i} \omega_{\text {im }}\right)$ we derive the following expressions for the real and imaginary parts of the frequency,

$\frac{\omega_{\mathrm{re}} L}{c_{\mathrm{Ac}}} \approx\left(\frac{m+1}{4}+(2 n-1)\right) \pi^{2}, n=1,2, \ldots$,

$-\frac{\omega_{\mathrm{im}} L}{c_{\mathrm{Ac}}} \exp \left(-\frac{\omega_{\mathrm{im}} L}{c_{\mathrm{Ac}}}\right) \approx \frac{1}{2 a / L\left(\rho_{\mathrm{l}} / \rho_{\mathrm{c}}-1\right)} \pi^{2}$.

In these equations we have a $\pi^{2}$ factor instead of the usual $\pi$ because of the normalisation in terms of $L=\pi r_{0}$ we have chosen for the magnetic field in Eq. (1): if we non-dimensionalize the frequency as $\omega r_{0} / c_{\text {Ac }}$ this extra factor disappears. As we can check, the approximations used to obtain Eqs. (23)-(25) are satisfied, since the imaginary part is large and tends to $-\infty$ when $a / L \rightarrow 0)$. The real part tends to a set of values that are not present in the Cartesian slab, but are multiples of $\pi^{2}$. Solving Eq. (23) directly confirms these results and shows that the real part decreases slowly from the value in Eq. (24) as $a / L$ increases. Notice also that as $a / L \rightarrow 0$ the imaginary part grows on an approximately logarithmic scale.

From Eq. (25) we deduce that there is no solution if the arcade is absent (both $a / L=0$ and $\rho_{1} / \rho_{\mathrm{c}}=1$ limits). The reason is we have used the boundary condition that there is no energy incoming from $r \rightarrow \infty$; but the only types of waves that can propagate in a semi-infinite system are freely propagating waves, which do not satisfy this criterion.

The values in Eq. (24) are not the expected ones for a Cartesian slab. However, in the limit of small $a / L$, the modes here are mainly trapped in the region under the arcade, a semicircular membrane of area $\pi r_{0}^{2}$. This point of difference is further discussed later in terms of the full dispersion relation and the spatial structure of its eigenfunctions.

\subsection{Magnetic ring}

As a limiting case, we consider a magnetic ring with rigid boundaries at $r=r_{0} \pm a$; we assume that the perturbed velocity $v_{r}$ vanishes at them. Then

$\bar{v}_{r}(r)=C_{1} r J_{m / 2}\left(k_{1}(r) r\right)+C_{2} r Y_{m / 2}\left(k_{1}(r) r\right)$.

The condition that the perturbation velocity vanishes on the boundary of the ring, so that $\bar{v}_{r}\left(r_{0} \pm a\right)=0$, leads to

$J_{m / 2}\left(\zeta_{-}^{1}\right) Y_{m / 2}\left(\zeta_{+}^{1}\right)-J_{m / 2}\left(\zeta_{+}^{1}\right) Y_{m / 2}\left(\zeta_{-}^{1}\right)=0$

where $\zeta_{ \pm}^{1}$ are defined in Eq. (20). We can invoke a series expansion of the zeros of this equation (Abramowitz \& Stegun 1967):

$\zeta_{-, n}^{1}=\beta+\frac{m^{2}-1}{\chi} \beta^{-1}+O\left(\beta^{-3}\right)$

with $\chi=\zeta_{+}^{1} / \zeta_{-}^{1}$ and

$\beta=\frac{n \pi}{\chi-1}=\frac{n(1-\pi a / L)^{2}}{4 a / L}$.

After some algebra, it leads to the result

$\frac{\omega_{n} L}{c_{\mathrm{Ac}}}=\frac{n \pi}{2 a / L \sqrt{\rho_{\mathrm{l}} / \rho_{\mathrm{c}}}}\left(1+\frac{2(a / L)^{2}\left(m^{2}-1\right)}{\pi n^{2}\left(1-\pi^{2}(a / L)^{2}\right)^{2}}+\ldots\right)$.

It is important to notice that (to first order) all the different types of Bessel functions have a similar value. The vertical modes $m=1$ are a special case in Eq. (30), since all the correction terms contain the term $\left(m^{2}-1\right)$ and so vanish. This can be shown to be exact by relating the Bessel functions of order $1 / 2$ with trigonometric functions (Abramowitz \& Stegun 1967), so Eq. (27) with $m=1$ becomes

$\sin \left(\zeta_{-}^{1}\right) \cos \left(\zeta_{+}^{1}\right)-\sin \left(\zeta_{+}^{1}\right) \cos \left(\zeta_{-}^{1}\right)=0$

with solutions (after using trigonometric relations)

$\zeta_{+}^{1}-\zeta_{-}^{1}=n \pi$

Using the definitions in Eq. (20) we obtain

$\frac{\omega_{n} L}{c_{\mathrm{Ac}}}=\frac{n \pi}{2 a / L \sqrt{\rho_{\mathrm{l}} / \rho_{\mathrm{c}}}}$

in agreement with Eq. (30) for $m=1$.

The result in Eq. (30) can be related directly to the case of a rigid Cartesian magnetic slab of length $L$ and width $2 a$. The dispersion relation for a slab turns out to be

$\frac{\omega_{n} L}{c_{\mathrm{Ac}}}=\frac{L}{\sqrt{\rho_{\mathrm{l}} / \rho_{\mathrm{c}}}} \sqrt{\frac{n^{2} \pi^{2}}{4 a^{2}}+\frac{m^{2} \pi^{2}}{L^{2}}}$,

where $n \pi /(2 a)$ is the effective wavelength in the $x$-direction and $m \pi / L$ is the wavelength in the $z$-direction, with $n$ and $m$ being the number of extrema in each direction and thus playing the same role as that in the magnetic ring. Using the Taylor expansion of the radical, we obtain

$\frac{\omega_{n} L}{c_{\mathrm{Ac}}}=\frac{n \pi}{2 a / L \sqrt{\rho_{\mathrm{l}} / \rho_{\mathrm{c}}}}\left(1+\frac{2(a / L)^{2} m^{2}}{\pi n^{2}}+\ldots\right)$,

to first order in $a / L$. The leading term in Eq. (35) is the same as the leading term in Eq. (30), but there are small modifications in the corrections due to the different geometries.

\subsection{Semi-circular membrane}

Finally, we explore the case of a semicircular domain with a rigid boundary at $r=r_{0}$. The solution to Eq. (13) is

$\bar{v}_{r}(r)=D_{1} r J_{m / 2}\left(k_{\mathrm{c}}(r) r\right)$

Imposing the condition $\bar{v}_{r}\left(r_{0}\right)=0$, we derive the simple dispersion relation:

$J_{m / 2}\left(\zeta_{\mathrm{c}}^{0}\right)=0$

with $\zeta_{\mathrm{c}}^{0}$ as defined earlier. All the zeros of the $J$ Bessel function are known to lie on the real axis if $m \geq 0$, which points that no leakage is present in this calculation. This is in accordance with the fact that here there is no extraction of energy from the medium: the system resembles a vibrating semi-circular membrane.

A simple expression can be obtained for the $n$th solution to Eq. (37) if we invoke the expansion of Bessel functions for large arguments:

$\frac{\omega_{n} L}{c_{\mathrm{Ac}}} \approx\left(\frac{m+1}{4}+(2 n-1)\right) \pi^{2}, n=1,2, \ldots$

This expression coincides with Eq. (24), pointing out that the modes in this limit are in fact the oscillations of a semi-circular membrane with rigid boundaries. 

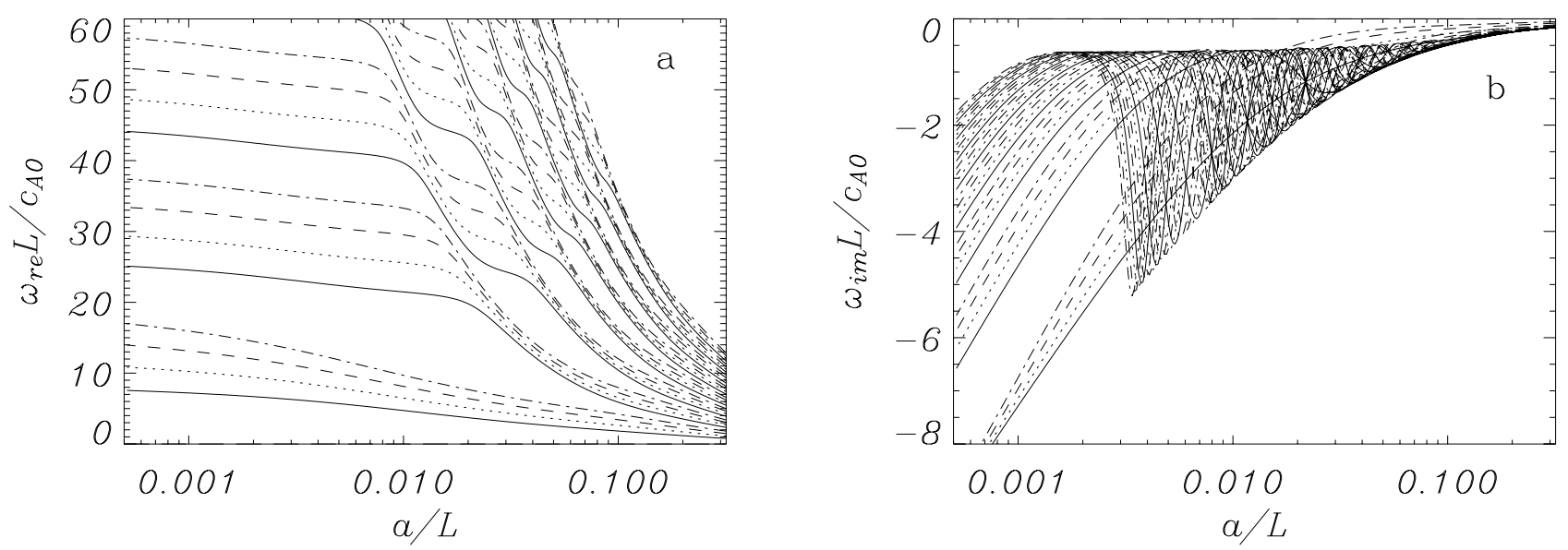

Fig. 2. Logarithmic plots of the a) real and b) imaginary parts of $\omega$ against the normalised radius for vertical modes ( $m=1$, solid lines), swaying modes ( $m=2$, dotted lines), and the two next families of modes ( $m=3$, dashed lines and $m=4$, dash-dotted lines). The density ratio has been fixed as $\rho_{\mathrm{l}} / \rho_{\mathrm{c}}=10$. Only the 8 lowest values for each family have been displayed.
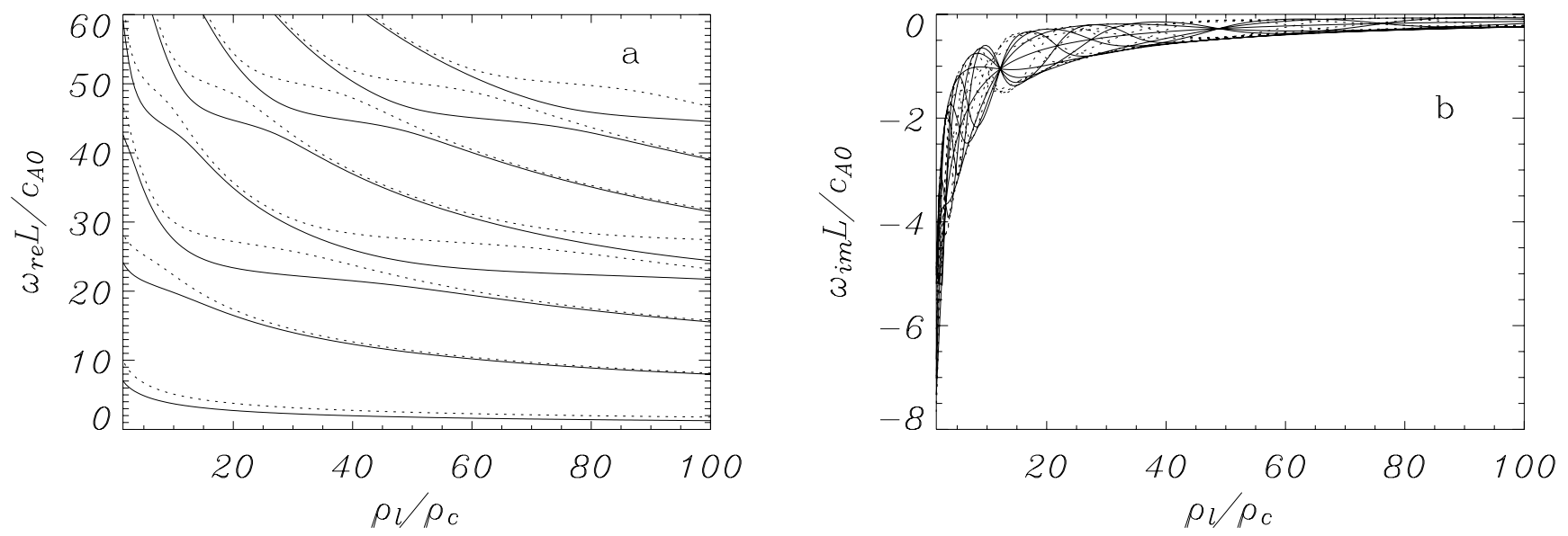

Fig. 3. Plots of the a) real and b) imaginary parts of $\omega$ against the density ratio for vertical modes $(m=1$, solid lines) and swaying modes $(m=2$, dotted lines). The width of the dense zone is fixed as $a / L=0.02$. Only the 8 lowest values for each family have been displayed.

\section{Results}

\subsection{Frequency dependence}

We now turn to a numerical solution of the full dispersion relation, Eq. (19). We start by plotting in Fig. 2 the solution to Eq. (19) in terms of the normalised width $a / L$ of the dense region. We have chosen the length of the arcade $L=\pi r_{0}$ as a unit. In this unit, a value of $a / L=1 / \pi$ implies that there is no evacuated part under the arcade, so in fact it becomes a semi-circular dense structure. Due to this, for $a / L \approx 1 / \pi$ the curves in Fig. 2 are equally spaced. For $a / L<1 / \pi$ we can see the presence of avoided crossings in the real part of $\omega$ for a certain range of values, and then the frequencies tend to the limiting values given in Eq. (24).

Regarding the imaginary part of $\omega$, we can also distinguish three regimes: for $a / L \approx 1 / \pi$ the modes are regularly spaced, but in the region of the avoided crossings all the harmonics have oscillations in frequency between two envelopes, which roughly correspond to the leakage of modes with structure only in the dense part of the arcade and modes with structure only in the cavity below the arcade. As $a / L \rightarrow 0$ the imaginary parts tend to infinity in a more regular pattern, in accordance with the result in Eq. (25). No modes can be trapped in this equilibrium without a density enhancement $(a / L=0)$, in contrast with the case of a straight slab between two photospheric surfaces, where all the medium can oscillate with zero transversal wavelength (no energy propagation from or towards infinity). This is the main reason why these modes cannot be compared to the ones of a homogeneous slab in the limit $a / L \rightarrow 0$, since the equilibria have different properties.

Notice that, except for small values of $a / L$, the imaginary parts of the frequency are small compared with the real parts, so the oscillations last for many periods before being attenuated. Also, the imaginary part is similar for all the harmonics while the real part is increased, meaning that the oscillations are damped in roughly the same time, but higher harmonics complete more periods in that amount of time.

In Fig. 3 we plot the dispersion relation for different values of density contrast. As the density ratio becomes large, the modes resemble those of a magnetic ring with rigid boundaries (no leakage), described in Sect. 3.2. It is evident that in this limit modes with different values of $m$ tend to the same frequencies, which correspond to the solutions to Eq. (27). On the other hand, when the density ratio is close to unity the imaginary parts grow in accordance with Eq. (25). In the expected range for coronal arcades and loops, say $2 \leq \rho_{\mathrm{l}} / \rho_{\mathrm{c}} \leq 20$, the imaginary part of the frequency is very sensitive to this ratio. This points out that the arcade is a better waveguide when the density contrast is increased, although the curvature always drives some leakage. 

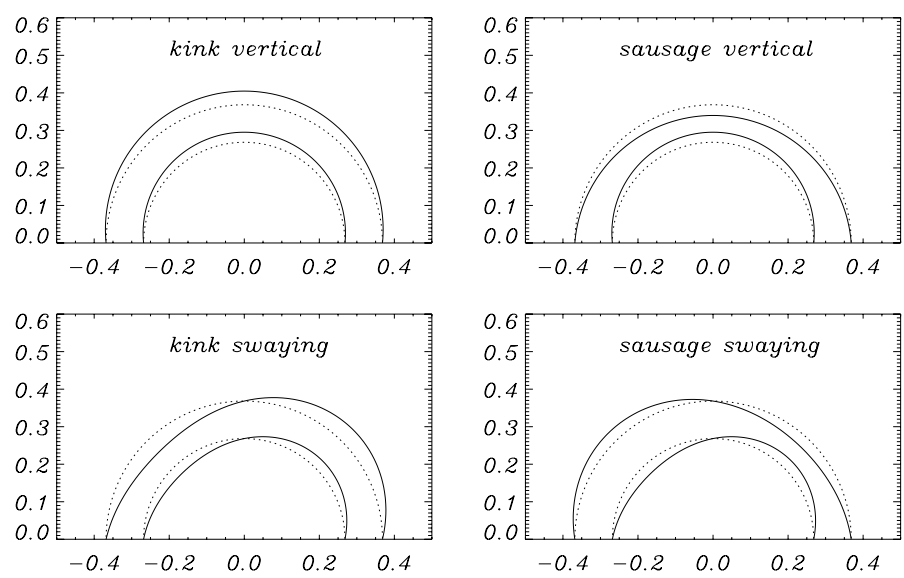

Fig. 4. Numerically determined oscillations of the region with dense plasma for various modes, at the initial instant (dotted) and after a quarter of the period (solid). Here $\rho_{\mathrm{l}} / \rho_{\mathrm{c}}=10$ and $a / L=0.05$, and the displacement is of amplitude $\left(r_{0}-a\right) / 10$.

\subsection{Spatial structure}

The oscillatory modes of an arcade can be labelled according to their $m$ number (which marks the number of extrema between the two footpoints). Modes with $m=1$ are modes in which the whole arcade is moving with the apex of the arcade moving vertically; these are the vertical modes. Modes with $m=2$ have a node at the arcade summit and they distort the arcade along its central axis; we name them swaying modes. They are longitudinal swaying modes since they lead apparently to motions in the direction of the equilibrium magnetic field. We refer to modes which lead to motions perpendicular to the magnetic arcade (in the $z$-direction) as transverse rocking modes; these modes are not discussed further here. Modes with higher values of $m$ are less important to our analysis and are referred to as fluting modes (by analogy with the fluting modes of a straight cylinder in the azimuthal direction).

There is also another important classification: kink and sausage modes (in accordance with the modes of a homogeneous tube). However, such a description is less evident here either from the perturbation equations or the dispersion relation as it is in the straight slab, but we can distinguish such characteristics by looking at the sign of the displacement in the boundaries $r=r_{0} \pm a$ : if it is the same, they are labelled as kink; if it is different, they are sausage modes.

In Fig. 4 we have represented the change in the position of the arcade boundaries for the different types of modes. Vertical modes displace the whole arcade mainly vertically, while in the swaying modes the displacement is more longitudinal. On the other hand, kink modes have very little compression, with the dense material being displaced as a whole and the centre being perturbed, while the sausage modes have marked compression and rarefaction and the centre of the arcade suffers little motion. Contrary to what happens in a straight slab, the sausage modes can have an axis displacement in this curved geometry, since in a slab the symmetry with respect to the axis allow us to classify the modes as kink or sausage, depending on their parity. In this geometry that parity is lost (since now there is a difference between the two parts of the arcade across the radial direction), but the idea of compression and rarefaction remains. Also, in a Cartesian geometry there is a change in the nature of the solution (hyperbolic functions instead of trigonometric, or modified Bessel functions instead of ordinary ones in the cylindrical straight tube); here the difference is more subtle.
It is also important to remember that all the modes are leaky and the solution grows exponentially away from the arcade. This is a well known feature in the straight cylinder (Cally 1986, 2003; Stenuit et al. 1998; Díaz et al. 2004) and can be directly related to the behaviour of the present problem. The interpretation of this fact is that the larger the distance from the arcade, the earlier the perturbation departed from the arcade and, since the term with the imaginary part of the frequency in Eq. (12) grows exponentially for reversed time, the bigger the amplitude in the density enhancement. The mode is unbounded and not square integrable, but we are looking for stationary states, and a leaky wave can only be stationary with this behaviour. The topic of leaky waves has recently attracted some vigorous discussion (Ruderman \& Roberts 2006; Cally 2006).

Spatial plots of $\bar{v}_{r}(r)$ are shown in Fig. 5 for a parameter set typical of coronal arcades. It is clear that functions grow exponentially far away from the arcade. In the zoomed region we can see the difference between kink and sausage modes. Notice also that as $r \rightarrow 0$ the functions scale as $r^{2}$, so the perturbed magnetic field in Eq. (6) is still bounded in this limit. For swaying and fluting modes the decay is stronger.

The other outstanding feature that appears in the dispersion relation is the presence of avoided crossings and the oscillations in the spatial structure. A closer look at this feature is provided in Fig. 6, were a spatial plot of the perturbed velocity is presented. The characteristics between the two modes are interchanged in the process. Avoided crossings are the result of two different types of modes interchanging their properties and this can happen because of coupling between different types of MHD modes (Oliver 1993), modes with spatial structure in different directions (Díaz et al. 2001) or modes with amplitude mainly in different regions (Donnelly et al. 2006). Here the avoided crossings are caused by the interaction of modes with different numbers of extrema in the inner region of the arcade (and therefore different imaginary parts of the frequency). As an extrema of the function approaches the dense region, the behaviour changes because of the continuity in the boundaries. These couplings cause oscillations in the imaginary part of the frequency (as seen in Figs. 2b and 3b); once the couplings disappear, the oscillations are stopped and the imaginary part of the frequency grows steadily as $a / L$ is diminished. Finally, note that the fundamental mode in each family does not have any avoided crossings, since its only extrema in $r \leq r_{0}+a$ lies always inside the dense region.

Another important feature is that the difference between kink and sausage modes is less marked once the zone of avoided crossings region is reached. In fact, these types of modes are only interlaced for the region of large $a / L$ where no avoided crossings are present, but once they appear it is not possible to discern which type of mode is obtained without plotting the spatial velocity structure. This again points to the fact that the distinction between sausage and kink modes is less important in an arcade geometry than for a straight field, since the symmetry between the inner and outer parts of the arcade with respect to the arcade axis is lost. However, the fundamental mode turns out to be always a kink mode and the next harmonic a sausage mode, but for small values of $a / L$ the next modes are also kink.

Finally, in the limit $a / L \rightarrow 0$ the extrema of the spatial structure are located in the coronal region under the arcade, with the dense rim acting as a confining surface. The difference between the modes of different order becomes the number of extrema in the radial direction displayed in the region $r \leq r_{0}-a$, in accordance with the results in Sects. 3.1 and 3.3. 

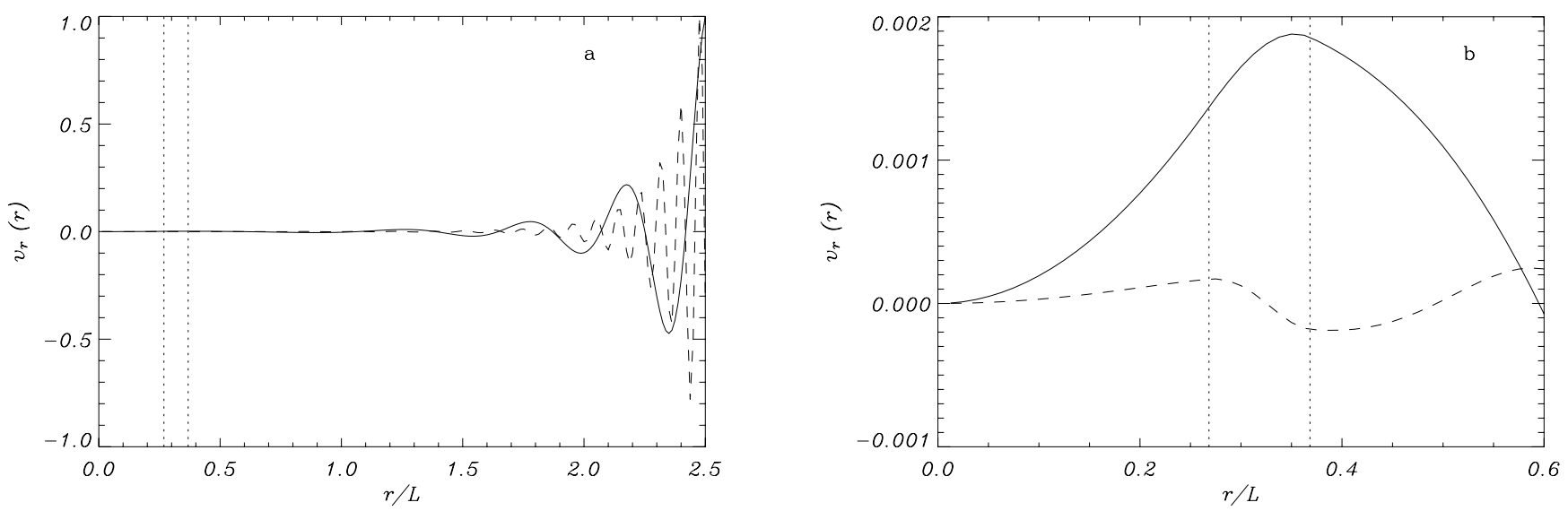

Fig. 5. a) Spatial structure of the fundamental vertical kink (solid line) and sausage (dashed line) modes for $a / L=0.05$ and $\rho_{\mathrm{l}} / \rho_{\mathrm{c}}=10$. The zone with dense plasma is indicated by the two vertical dotted lines. b) Zoom in the region of the dense plasma.
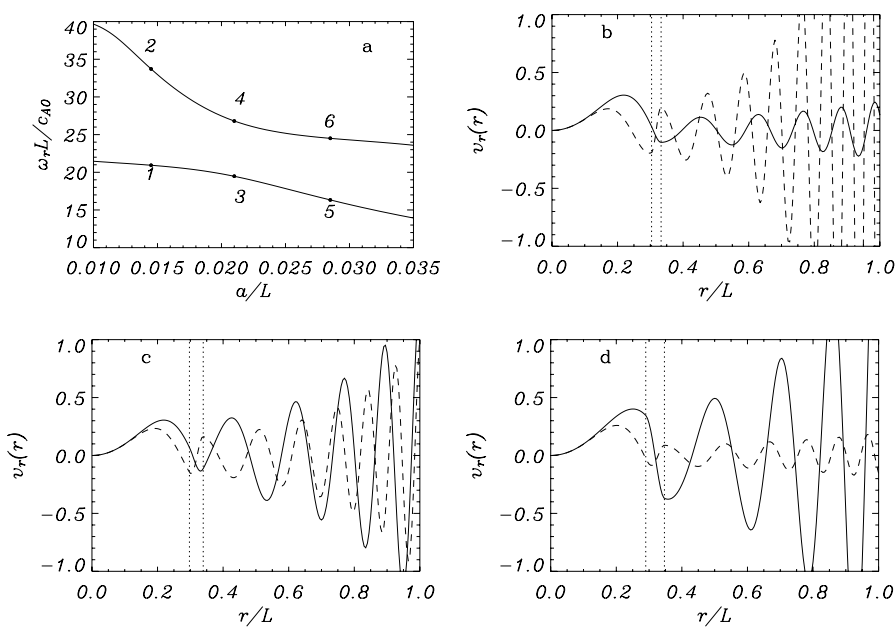

Fig. 6. Avoided crossings: a) zoom of the real part of the dispersion relation in Fig. 2 for the vertical modes $\left(\rho_{1} / \rho_{\mathrm{c}}=10\right)$; panels b), c) and d) are the spatial plot of the perturbed velocity (in arbitrary units) for the modes with $a / L=0.0145$ (points 1 and 2), $a / L=0.021$ (points 3 and 4 ) and $a / L=0.0285$ (points 5 and 6 ), respectively. In these panels the mode with lower frequency is plotted in a solid line and the mode with higher frequency in a dashed line. The limit of the dense zone is marked by a pair of vertical dotted lines.

\subsection{Comparison with a straight magnetic slab}

It is instructive to compare results for the curved arcade with the equivalent ones of a straight magnetic slab (Edwin \& Roberts 1982; see also Verwichte et al. 2005). We have already seen in Sect. 3.2 that in the limit of a very dense structure (effectively ruling out the surrounding medium) the differences are only geometrical factors, though this limit is far from typical coronal values.

To compare with the results of a homogeneous Cartesian slab we notice that the azimuthal direction plays the role of the $z$-direction in a slab, and hence, the $m$ number here is equivalent to the number of extrema in the $z$-direction, since $k_{z}^{\text {cart }}=m \pi / L$. It is known that the Cartesian slab has only one trapped mode for each family (the fundamental one), while the harmonics are leaky and have an imaginary part due to the coronal leakage (although some of these modes can be trapped too for values of $a / L$ of the order of unity). With this in mind, we can plot together the results for the two different geometries. In Fig. 7 we have plotted the vertical modes and their Cartesian counterparts with just one extrema in the $z$-direction. Similar plots can be produced for the swaying and fluting modes.

Figure $7 \mathrm{a}$ reveals that for sufficient large $a / L$ there are only small corrections, and kink and sausage modes correspond to consecutive modes of the curved arcade. However, once the avoided crossings appear the modes are no longer close to their Cartesian counterparts; moreover the fundamental kink mode (which is always trapped in the Cartesian model) is different in the two geometries.

In fact, the Cartesian modes correspond to one of the types of modes which are affected by the avoided crossings. There are no avoided crossings in the Cartesian slab. This difference can be explained by the fact that avoided crossings are caused by interaction between typical kink and sausage modes and other modes which have extrema in the inner coronal region $r \leq r_{0}-a$. Since in a Cartesian geometry both coronal regions are identical, there is no trapping of a mode under the structure and avoided crossings do not happen. The modes of the curved arcade seem to outline one of the types of modes which suffer the avoided crossings. Therefore, in the region of small $a / L$, where these modes dominate, the two geometries behave completely differently (this is not evident in the present plot, unless a logarithmic scale is introduced): the curved structure is similar to a semi-circular membrane, while the Cartesian structure resembles a rectangular membrane.

Regarding the complex part of the frequency (Fig. 7b), some differences are evident between the two models. However, the most interesting point is that the modes of the curved slab have less attenuation, by a factor 2, than their Cartesian counterparts. This indicates that the curved structure is a slightly better waveguide than the straight slab, although it is not capable of confining the fundamental mode, since curvature seems to enhance that leakage. This can be explained by noticing that the Cartesian slab leaks in both the negative and positive $x$-directions, while the arcade leaks only towards $r \rightarrow \infty$, and therefore the decay rate is roughly halved.

\subsection{Periods and damping times}

We now give some numerical estimates following the values reported for a solar coronal arcade by Verwichte et al. (2004), namely, a field line of length $L=200 \mathrm{Mm}$ and an Alfvén speed of $c_{\mathrm{Ac}} \approx 1000 \mathrm{~km} \mathrm{~s}^{-1}$ (so $B_{0} \approx 10 \mathrm{G}$ ); we take $a / L=0.025$ and $\rho_{\mathrm{l}} / \rho_{\mathrm{c}}=10$. 

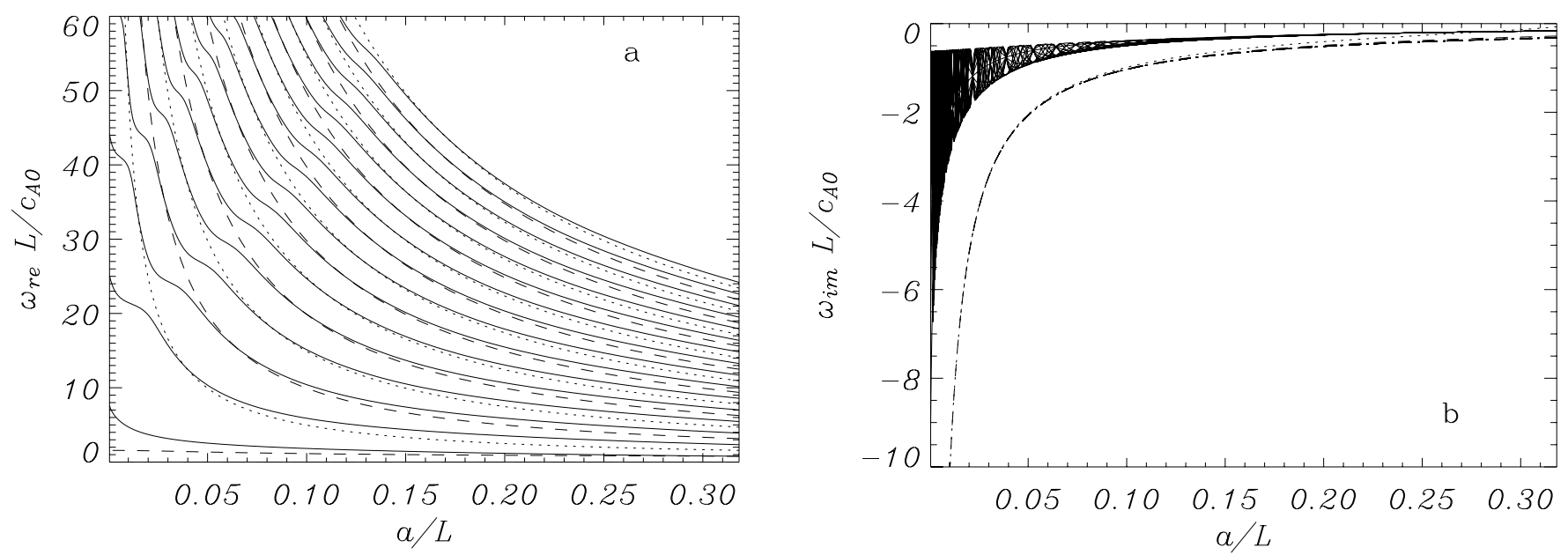

Fig. 7. Plots of a) real and $\mathbf{b}$ ) imaginary parts of $\omega$ against the half-width $a / L$ of the dense zone for vertical modes $(m=1)$. The solid lines correspond to the results of the curved arcade, while the dashed and dotted lines correspond to the kink and sausage modes, respectively, of the straight Cartesian slab. Notice that the fundamental mode for the Cartesian slab is trapped for all the values of $a / L$, so its imaginary part is identically zero in panel $\mathbf{b}$ ).

Table 1. Periods $P$ and damping times $\tau$ of the first four vertical and swaying modes for $L=200 \mathrm{Mm}, c_{\mathrm{Ac}}=1000 \mathrm{~km} \mathrm{~s}^{-1}, a / L=0.025$ and $\rho_{\mathrm{l}} / \rho_{\mathrm{c}}=10 . N_{\text {inner }}$ and $N_{\text {dense }}$ give the number of extrema in the spatial structure of the perturbed velocity in the inner coronal part and in the dense part, respectively.

\begin{tabular}{cccc|ccc}
\hline \hline Type & sym. & $N_{\text {inner }}$ & $N_{\text {dense }}$ & $P(\min )$ & $\tau(\min )$ & $\tau / P$ \\
\hline vertical & kink & 0 & 1 & 6.15 & 3.13 & 0.51 \\
swaying & kink & 0 & 1 & 4.46 & 4.26 & 0.96 \\
vertical & saus & 0 & 2 & 1.17 & 2.45 & 2.09 \\
swaying & saus & 0 & 2 & 1.09 & 2.11 & 1.94 \\
vertical & saus & 1 & 2 & 0.83 & 4.30 & 5.17 \\
swaying & saus & 1 & 2 & 0.73 & 5.98 & 8.21 \\
vertical & kink & 0 & 3 & 0.57 & 2.15 & 3.77 \\
swaying & kink & 0 & 3 & 0.54 & 2.04 & 3.89 \\
\hline
\end{tabular}

We have calculated the resulting periods $P=2 \pi / \omega_{\text {re }}$ and damping times $\tau=1 / \omega_{\text {im }}$ from Eq. (19). The results are gathered in Table 1. Classifying the modes is quite complex, so we have also included information about the number of extrema of the velocity in each region. It is clear from Fig. 2 that avoided crossings have already taken place for the value of $a / L$ used in the Table, and this is the reason for the occurrence of two consecutive sausage modes (the second with an extrema in the inner coronal region). We can also see in Fig. 7 that the corresponding modes for the Cartesian slab are somewhat different (for example, the fundamental kink mode has a period of 7.04 min compared with the $6.15 \mathrm{~min}$ in the arcade; differences are larger for other modes).

The most important feature is that the periods are in broad accordance with the values given in Verwichte et al. (2004) (despite the presence there of a horizontal displacement). Also, the fundamental mode is damped after a few periods. The ratio $\tau / P$ is not dependent on the values of $L$ and $c_{\mathrm{Ac}}$, but only on the dimensionless ratios $a / L$ and $\rho_{\mathrm{l}} / \rho_{\mathrm{c}}$. In Fig. 8 we present the dependence of $\tau / P$ for various values of the arcade density; $\tau / P$ depends strongly on the density ratio, and so can be used as a tool for coronal seismology. Figure 8 can be compared with the observational results for coronal loops in Aschwanden et al. (2003), which are also compared with damping by resonant absorption in a cylindrical flux tube. Coronal leakage gives a $\tau / P$ ratio that is comparable with the observed values. However, the trend is

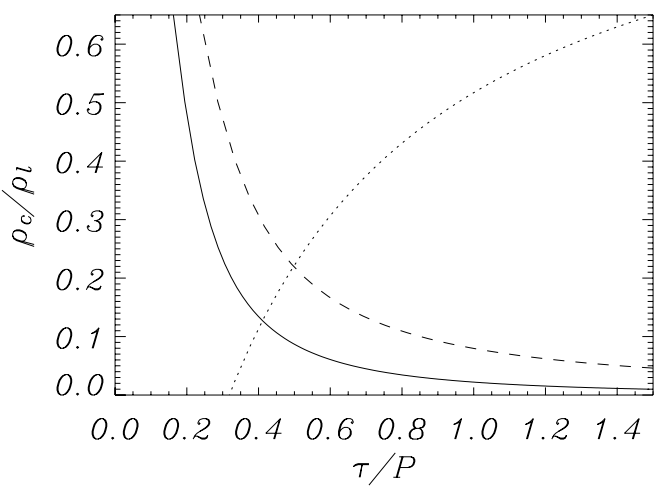

Fig. 8. Density ratio $\rho_{\mathrm{c}} / \rho_{\mathrm{l}}$ against the ratio of the damping time to the period $\tau / P$ for the fundamental vertical mode ( $m=1$, solid line) and the fundamental swaying mode $(m=2$, dashed line) for $a / L=0.025$. The results due to resonant absorption have been overplotted in a dotted line.

different, since for coronal leakage the higher the density contrast the better the confinement, while for resonant absorption the opposite is true, since higher density contrast means steeper gradients (the main effect depends upon the scale $l$ of the inhomogeneity). Higher harmonics damp on a similar timescale, but since their periods are considerably shorter these modes will be much harder to detect, despite lasting for more periods before being damped.

The particular parameters we have used are in the regime in which our model is not similar to the modes of the Cartesian slab, but rather is in the region of the dispersion diagrams where interactions and avoided crossings take place.

\section{Discussion and conclusions}

We have studied the radially polarised modes of a semi-circular curved arcade with a force-free field and constant plasma density zones. The resulting Alfvén speed profile decreases with $r$. There are several interesting conclusions:

1. For our choice of equilibrium, there are no trapped modes; even the fundamental mode is leaky for all parameters. 
However, trapped modes are possible for other density profiles (Verwichte et al. 2005).

2. Various modes arise, including vertical, swaying and fluting modes. The swaying modes are a new type of mode not present in the straight slab or tube configuration studied in Edwin \& Roberts $(1982,1983)$.

3 . For thick arcades $(a / L$ large $)$, there are few differences between these modes and those of a straight Cartesian slab, though the decay rate of a mode is half the corresponding rate in a slab.

4. For thin arcades $(a / L$ small $)$, the modes are similar to those of a semi-circular membrane, corresponding to the oscillations of the cavity under the dense arcade. Explicit expressions are derived for the period and damping times of these modes (Eqs. (24) and (25)).

5. For intermediate values of $a / L$, avoided crossings take place between the two types of modes. Solar coronal arcades have parameters in this region of the dispersion diagrams.

6. The oscillations typically last $2-5$ periods, being damped by leakage through the coronal surroundings. The exact damping time depends on the density contrast between the arcade and the surroundings.

The presence of various modes is expected on geometrical grounds, and it has been discussed in some observational (Wang $\&$ Solanki 2004) and numerical (Selwa et al. 2005) studies. However, an analytical discussion has been lacking. In fact, in a three dimensional structure there are also rocking (or horizontal) modes, for which the structure is displaced in the transversal direction (the $z$-direction in Fig. 1), but the detailed structure of rocking modes requires a separate study. In a curved loop, all three types of mode (vertical, swaying and rocking) are present with distinct frequencies, but if the structure is straightened they become degenerate. A curved loop would behave different from an arcade in the limit of small radius, since here there is a cavity under the arcade, while the material under a curved loop can be displaced around it. Further work is needed to study the modes in a curved coronal loop, but the arcade indicates the presence of the swaying modes and provides some guidance.

Another striking result is the absence of purely trapped modes in the arcade. In fact, observations show that all loop modes are damped in a few periods. This absence of trapped modes is in accordance with Verwichte et al. (2005), since our equilibrium corresponds to the special case when with their parameter $\alpha$ is zero and, as they point out, this selection of $\alpha$ leads to leakage. Moreover, the damping rates are in accordance with observational reports for arcades (Verwichte et al. 2004). The oscillations in other structures, such as coronal loops or prominences, are also damped in a few periods. These results are an indication that coronal leakage may be important for the understanding of the observed damping rate. Finally, notice also that the damping rate contains information about the equilibrium and plasma properties (see Eq. (25), for example), so it can be used in addition to the period as a tool for coronal seismology.

Acknowledgements. The authors thank Dr. A. N. Wright and Prof. Y. D. Zhugzhda for useful comments and discussion. AJD acknowledges support from PPARC on the St Andrews Solar Theory Rolling Grant. The work of T.Z. was partially supported by the Spanish MCyT grant AYA2003-00123.

\section{References}

Abramowitz, M., \& Stegun, I. A. 1967, Handbook of Mathematical Functions (Dover)

Allan, W., White, S. P., \& Poulter, E. M. 1986a, Planet Space Sci., 34, 371

Allan, W., White, S. P., \& Poulter, E. M. 1986b, Planet Space Sci., 34, 1189

Andries, J., Goossens, M., \& Arregui, I. 2005, ApJ, 624, L57

Andries, J., Goossens, M., Hollweg, J. V., Arregui, I., \& Van Doorsselaere, T. 2005, A\&A, 430, 1109

Aschwanden, M. J. 2004, Physics of the corona (Springer Praxis Books in Geophysical Sciences)

Aschwanden, M. J., Nightingale, R. W., Andries, J., Goossens, M., \& Van Doorsselaere, T. 2003, ApJ, 598, 1375

Brady, C. S., \& Arber, T. D. 2005, A\&A, 438, 733

Cally, P. S. 1986, Sol. Phys., 103, 277

Cally, P. S. 2003, Sol. Phys., 217, 95

Cally, P. S. 2006, Sol. Phys., 223, 79

Díaz, A. J. 2004, Ph.D. Thesis, Universitat de les Illes Balears

Díaz, A. J., Oliver, R., Erdélyi, R., \& Ballester, J. L. 2001, A\&A, 379, 1083

Díaz, A. J., Oliver, R., Ballester, J. L., \& Roberts, B. 2004, A\&A, 424, 1055

Donnelly, G. R., Díaz, A. J., \& Roberts, B. 2006, A\&A, accepted

Edwin, P. M., \& Roberts, B. 1982, Sol. Phys., 76, 239

Edwin, P. M., \& Roberts, B. 1983, Sol. Phys., 88, 179

Goedbloed, J. P. 1983, Lecture Notes on Ideal Magnetohydrodynamics, Rijnhuizen Report

Goossens, M. 1991, in Advances in Solar System Magnetohydrodynamics, ed. E. R. Priest, \& A. W. Hood (Cambridge Univ. Press), 137

Heyvaerts, J., \& Priest, E. R. 1983, A\&A, 117, 220

Lee, D. H., \& Lysak, R. L. 1989, J. Geophys. Res., 94,17097

McEwan, M., Donnelly, G. R., Díaz, A. J., \& Roberts, B. 2006, A\&A, accepted Nakariakov, V. N., \& Verwichte, E. 2005, Living Rev. Solar Phys., 2, http: //www. livingreviews.org/lrsp-2005-3

Oliver, R., Ballester, J. L., Hood, A. W., \& Priest, E. R. 1993, ApJ, 409, 809

Roberts, B., Edwin, P. M., \& Benz, A. O. 1984, ApJ, 279, 857

Ruderman, M. S., \& Roberts, B. 2006, J. Plasma Phys., 72, 285

Selwa, M., Murawski, K., Solanki, S. K., Wang, T. J., \& Tóth, G. 2005, A\&A, 440,385

Smith, J. M., Roberts, B., \& Oliver, R. 1997, A\&A, 317, 752

Stenuit, H., Keppens, R., \& Goossens, M. 1998, A\&A, 331, 392

Van Doorsselaere, T., Debosscher, A., Andries, J., \& Poedts, S. 2004, A\&A, 424, 1065

Verwichte, E., Nakariakov, V. M., Ofman, L., \& Deluca, E. E. 2004, Sol. Phys., 223, 77

Verwichte, E., Nakariakov, V. M., \& Cooper, F. C. 2005, A\&A, 430, L65

Verwichte, E., Foullon, C., \& Nakariakov, V. M. 2006, 446, 1139

Wang, T. J., \& Solanki, S. K. 2004, A\&A, 421, L33

Wright, A. N. 1992, J. Geophys. Res., 97, 6429 